\title{
Synthesis of submicron particles of a stereoregular polyolefin by catalysis in aqueous dispersion
}

\author{
Amaia Bastero and Stefan Mecking* \\ Supporting information
}

General considerations. All manipulations of air-sensitive compounds were performed using standard Schlenk techniques under argon atmosphere.

NMR spectra were recorded with either a Bruker ARX 300, Bruker Avance DRX 600 or Varian INOVA-400 spectrometer. ${ }^{1} \mathrm{H}$ and ${ }^{13} \mathrm{C}$ NMR chemical shifts were referenced to ${ }^{1} \mathrm{H}$ and ${ }^{13} \mathrm{C}$ NMR signals of the deuterated solvents, respectively. High temperature NMR measurements were performed in $1,1,2,2$-tetrachloroethane- $d_{2}$ at $130^{\circ} \mathrm{C}$ and at $100^{\circ} \mathrm{C}$. IR spectra were recorded on a FT-IR Bruker Vector 22 spectrometer using $\mathrm{KBr}$ pellets. Dynamic light scattering (DLS) on dispersions was performed on a Malvern Nano-ZS ZEN 3600 particle sizer $\left(173^{\circ}\right.$ back scattering). Differential scanning calorimetry (DSC) was performed on either a Perkin-Elmer DSC 7, a Pyris 1 DSC or a Netzsch DSC 204 F1 at a heating rate of $10 \mathrm{~K} / \mathrm{min}$. The polymers were investigated in the temperature range $25-230^{\circ} \mathrm{C}$. DSC data reported are second heats. Transmission electron microscopy (TEM) was carried out on a LEO 912 Omega apparatus using an acceleration voltage of $120 \mathrm{kV}$. Samples were prepared by applying a drop of the dispersion to a carbon coated grid. Molecular weight determination was done with a PL GPC-220 device using a Mixed B column with a refractive index detector, in trichlorobenzene at $160^{\circ} \mathrm{C} v s$. polyethylene standards.

Materials. Ruthenium(III) tris-2,4-pentanedionate (Merck) and triphenylphosphine (Aldrich) were used without further purification. Hydrogen (99.999 \% purity) was purchased from Messer-Griesheim and used as received. Toluene was distilled from sodium under argon. 1,2polybutadiene latices were synthesized according to reference [1] and were degassed using an argon stream before hydrogenation.

Hydrogenation procedure. The hydrogenation was carried out in a mechanically stirred 285 $\mathrm{mL}$ high-pressure reactor equipped with a heating/cooling block controlled by a thermocouple dipping into the reaction mixture. The 1,2-polybutadiene dispersion (40 mL; 4-6\% solids content), previously degassed, was introduced in the reactor under an argon atmosphere. The catalyst was prepared by mixing ruthenium(III) tris-2,4-pentanedionate and triphenylphosphine, in a 1:4 molar ratio, in $2 \mathrm{~mL}$ of toluene. After stirring for about 5 minutes the red solution obtained was transferred to the reactor. The reactor was flushed and pressurized with hydrogen, while rapidly heating to $125^{\circ} \mathrm{C}$ under stirring $(1000 \mathrm{rpm})$. After the specified reaction time, the reaction was stopped by cooling and releasing the pressure. The polymer dispersion was taken out of the reactor with a syringe and further analyzed. For NMR, IR, DSC and GPC analysis the dispersion was added to methanol under stirring and the precipitated polymer was filtrated, washed with methanol and dried at $50^{\circ} \mathrm{C}$ under vacuum overnight.

[1] a) Henderson, J. N.; Donbar, K. W.; Barbour, J. J.; Bell, A. J. (Goodyear) US Patent 4429085, 1984. b) Ono, H.; Matsumura, Y.; Okuya, E. (JSR) EP Patent 0152175, 1985. 

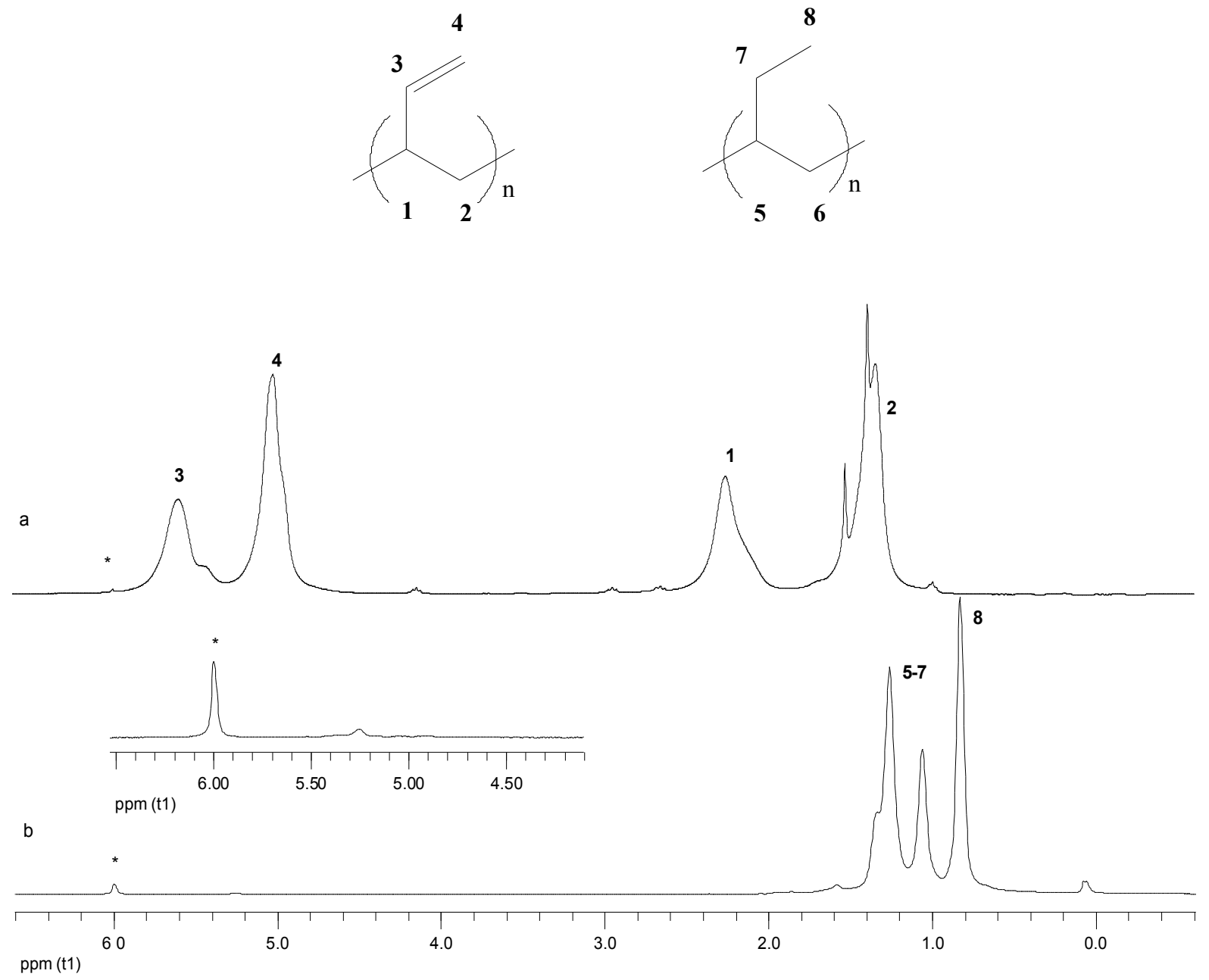

Figure S1. ${ }^{1} \mathrm{H}$ NMR spectra of (a) 1,2-polybutadiene; (b) fully hydrogenated polybutadiene; *: solvent signal (tetrachloroethane). The small signal at $5.25 \mathrm{ppm}$ can been assigned to trisubstituted double bonds $\left(\mathrm{R}_{2} \mathrm{C}=\mathrm{CHMe}\right)$ formed by isomerization (cf. Rossi, A.; Odian, G.; Zhang, J. Macromolecules 1995, 28, 1739-1749.). Integration shows $>99 \%$ degree of hydrogenation. 

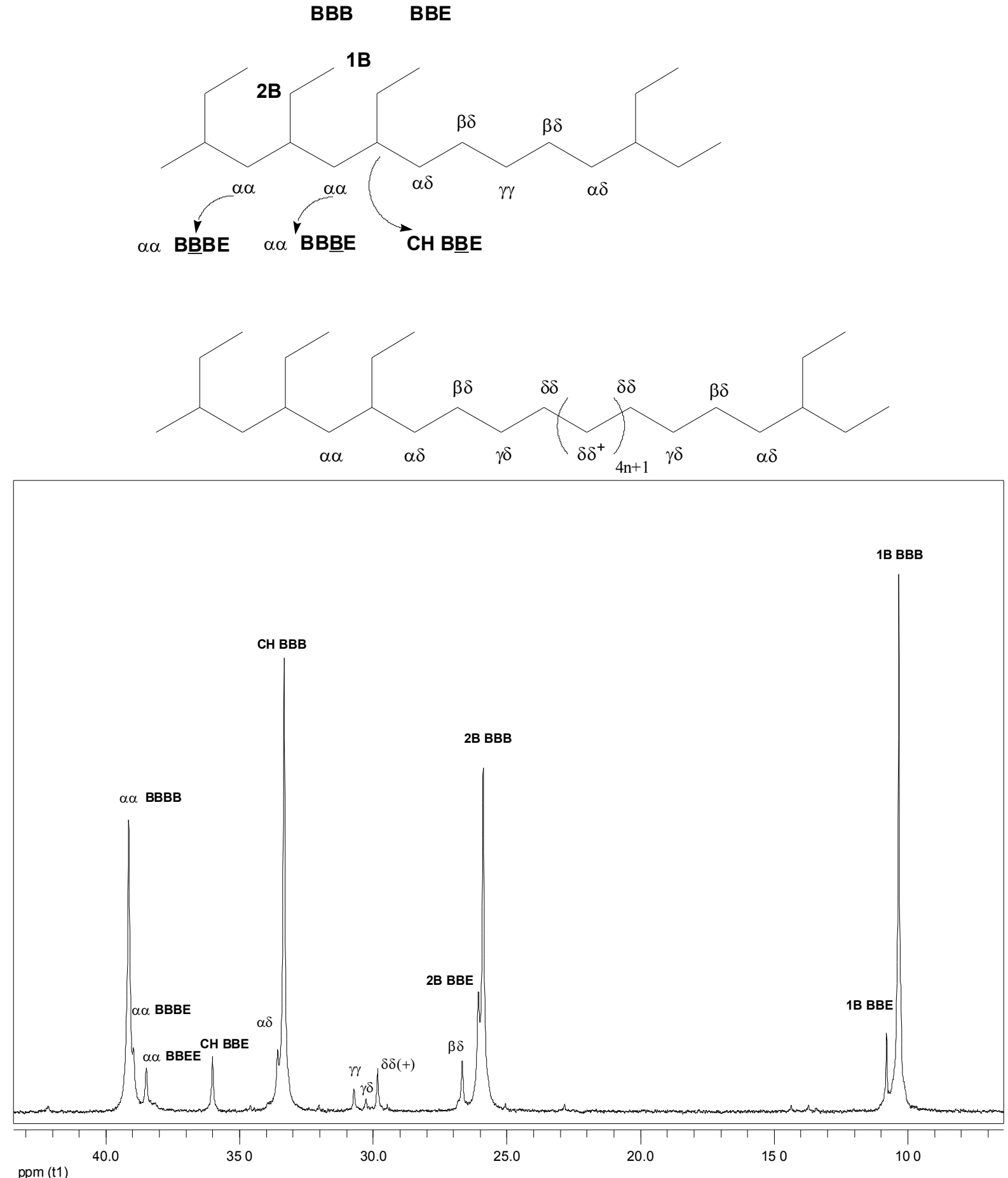

Figure S2. ${ }^{13} \mathrm{C}$ NMR of a $100 \%$ hydrogenated polymer (hydrogenation conditions: $[\mathrm{C}=\mathrm{C}] /$ [Ru]: 500, $125^{\circ} \mathrm{C}, 75$ bar hydrogen). Assignments according to Sahoo, S. K.; Zhang, T.; Reddy, D. V.; Rinaldi, P. L.; McIntosh, L. H.; Quirk, R. P. Macromolecules 2003, 36, 401728 and also confirmed by DEPT experiments. 

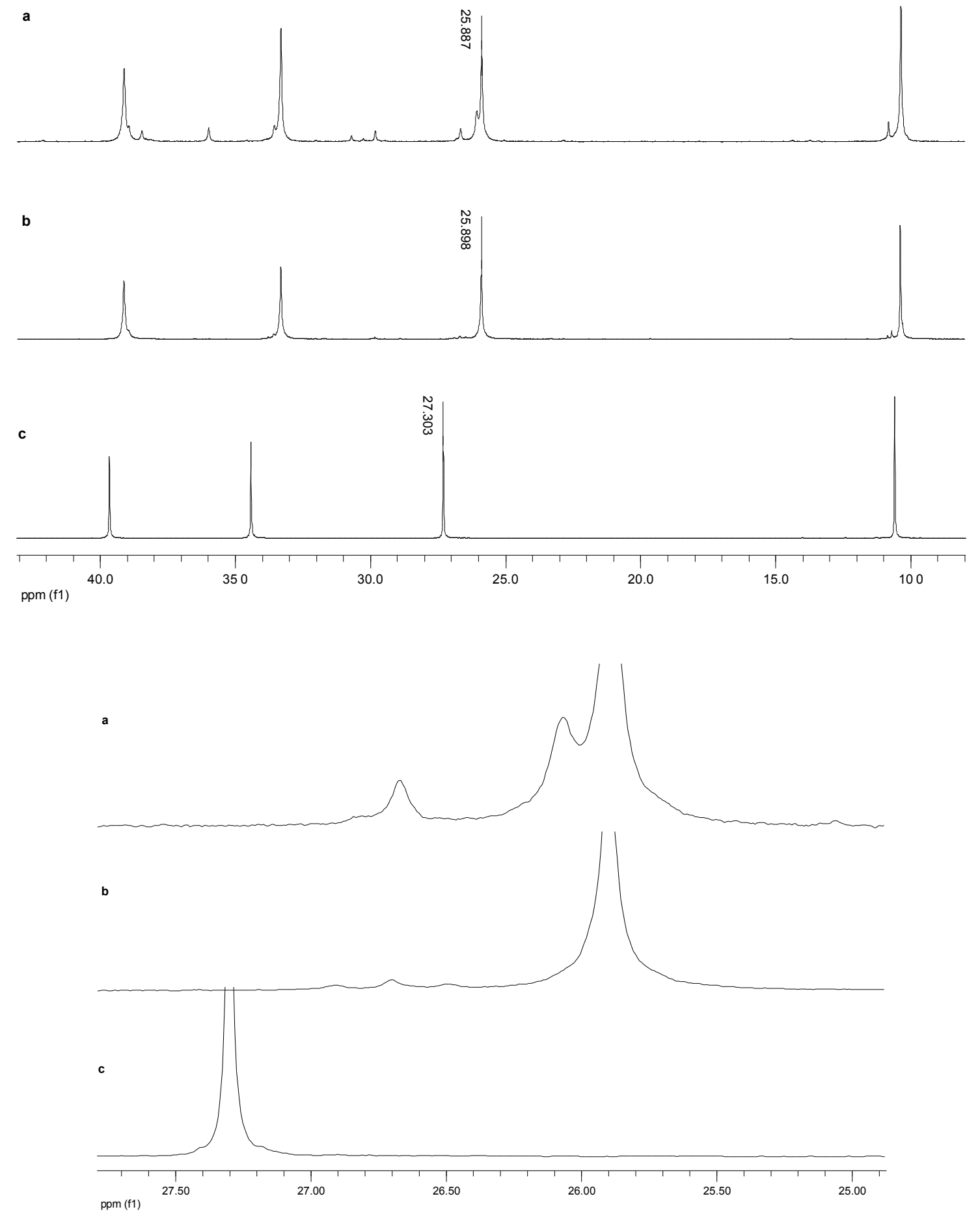

Figure S3. ${ }^{13} \mathrm{C}$ NMR spectra for tacticity determination: (a) $>99 \%$ hydrogenated polymer; (b) syndiotactic poly(1-butene) (prepared according to Asanuma, T.; Nishimori, Y.; Ito, M.; Uchikawa, N.; Shiomura, T. Polym. Bull. 1991, 25, 567-70 with [\{(Flu $\left.\left.\mathrm{CMe}_{2}(\mathrm{Cp})\right\} \mathrm{ZrCl}_{2}\right] /$ MAO; Zr: $6 \mu \mathrm{mol}$; Al/Zr: 500; 30 ${ }^{\circ} \mathrm{C} ; 2 \mathrm{~h}$ ); (c) isotactic poly(1-butene). Bottom: enlargement of range 24.9 to $27.8 \mathrm{ppm}$. 


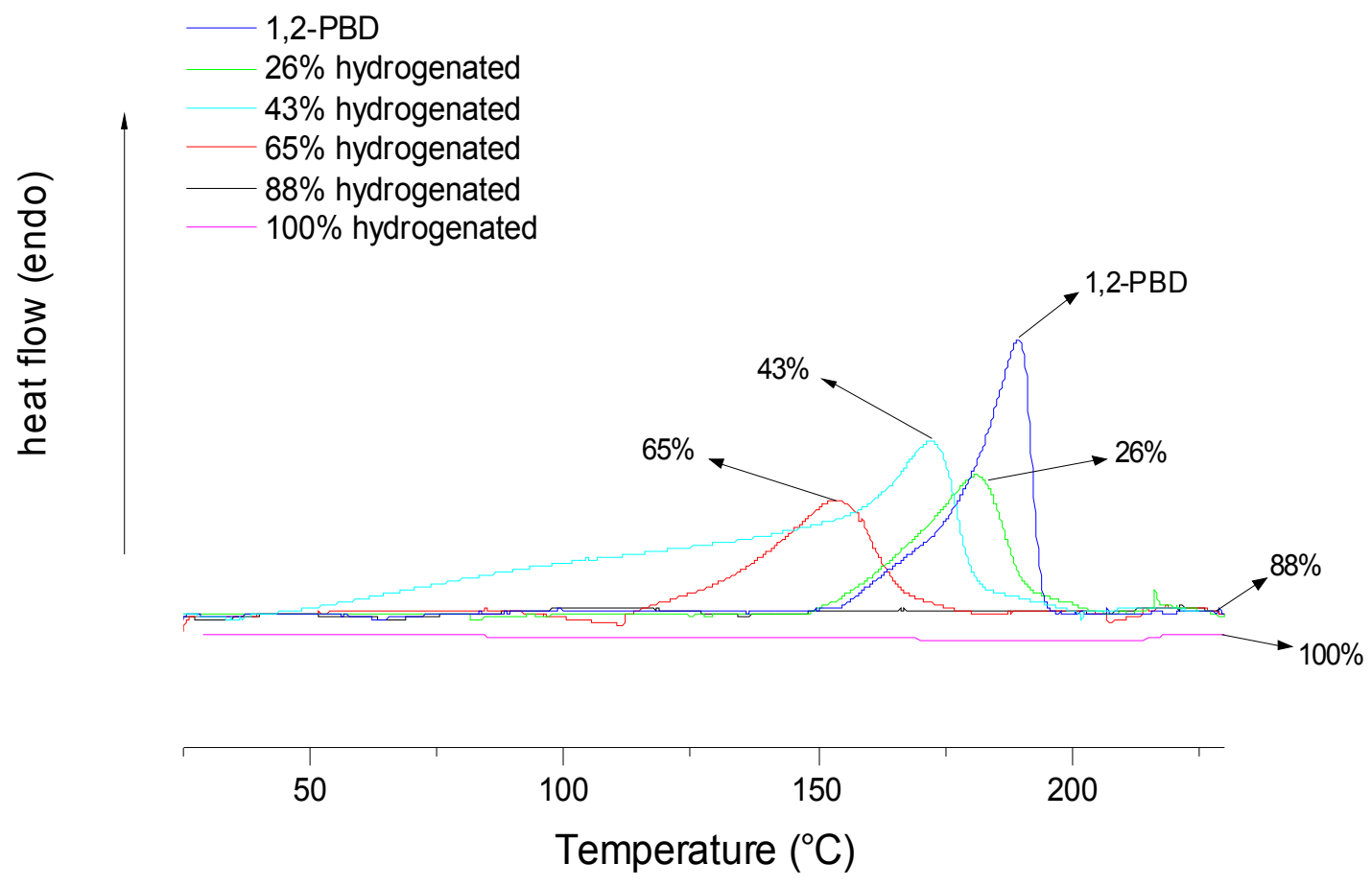

Figure S4. Comparative DSC measurements performed on isolated bulk polymers obtained from the different latices. Second heating curves are shown; heating rate: $10^{\circ} \mathrm{C} \mathrm{min}^{-1}$. 


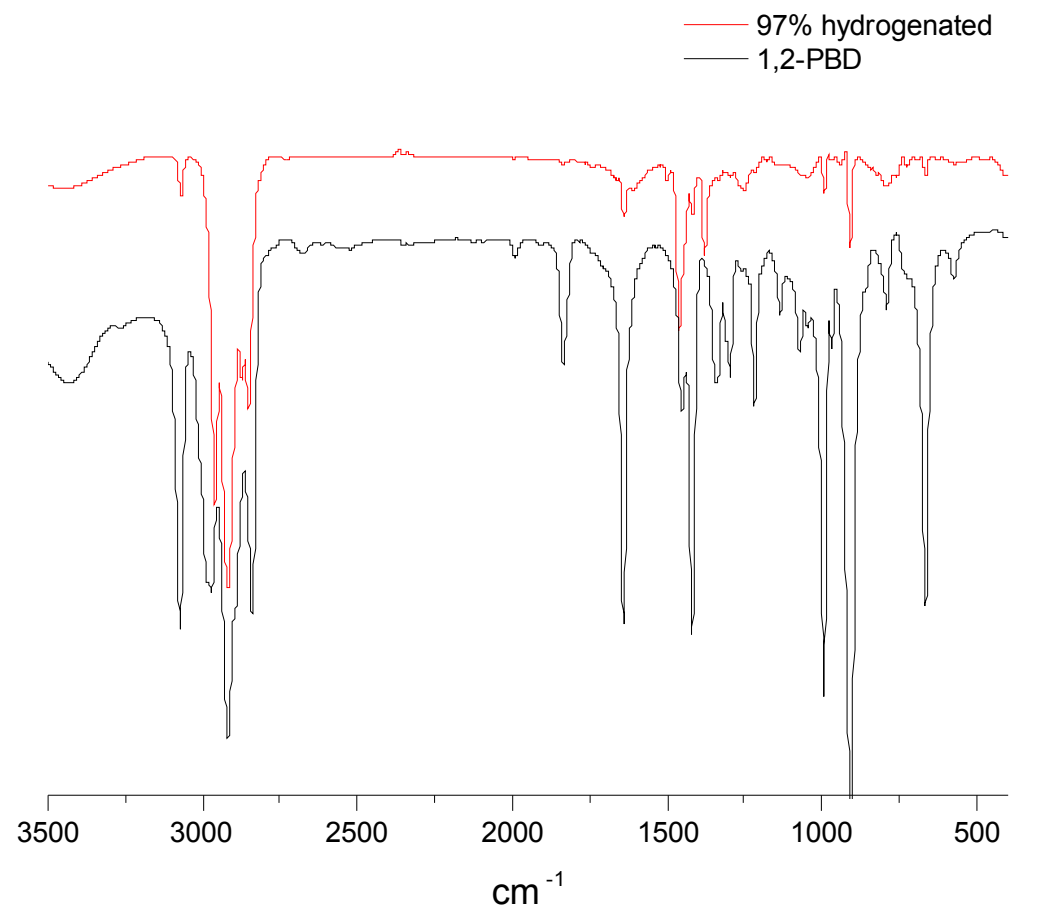

Figure S5. Comparative IR spectra performed on isolated bulk polymers: (a) $97 \%$ hydrogenated; (b) 1,2-polybutadiene. Spectrum (a) shows diminishing of the typical absorption bands of polybutadiene, at $911 \mathrm{~cm}^{-1}$ for 1,2-units, $737 \mathrm{~cm}^{-1}$ for cis-1,4 units and $967 \mathrm{~cm}^{-1}$ for trans-1,4 units, together with appearance of the strongest bands in the 2900-2800 and 1460-1360 $\mathrm{cm}^{-1}$ ranges. For comparison see: Ishioka, T.; Wakisaka, H.; Kanesaka, I.; Nishimura, M.; Fukasawa, H. Polymer 1997, 38, 2421-30. 
(a)

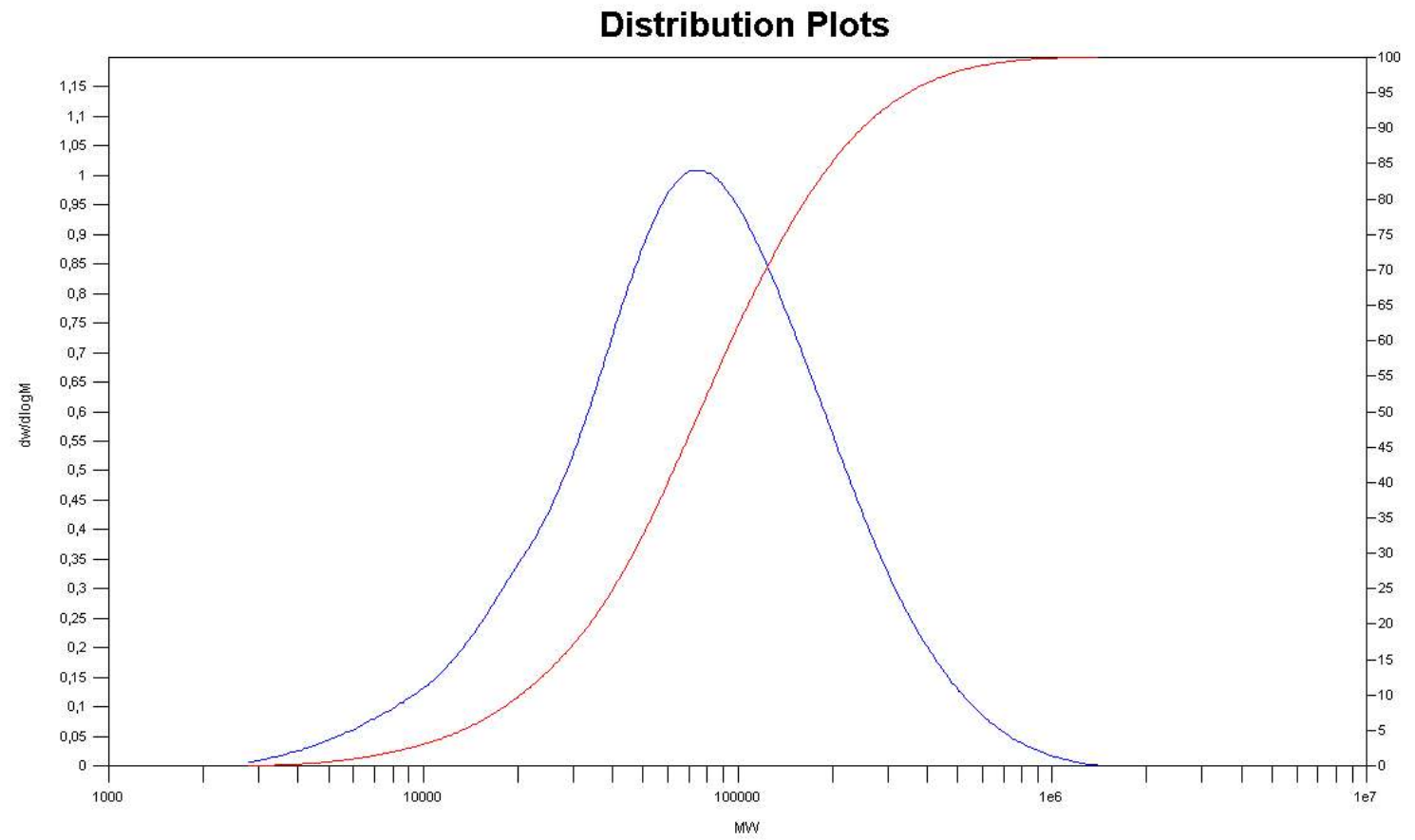

(b)

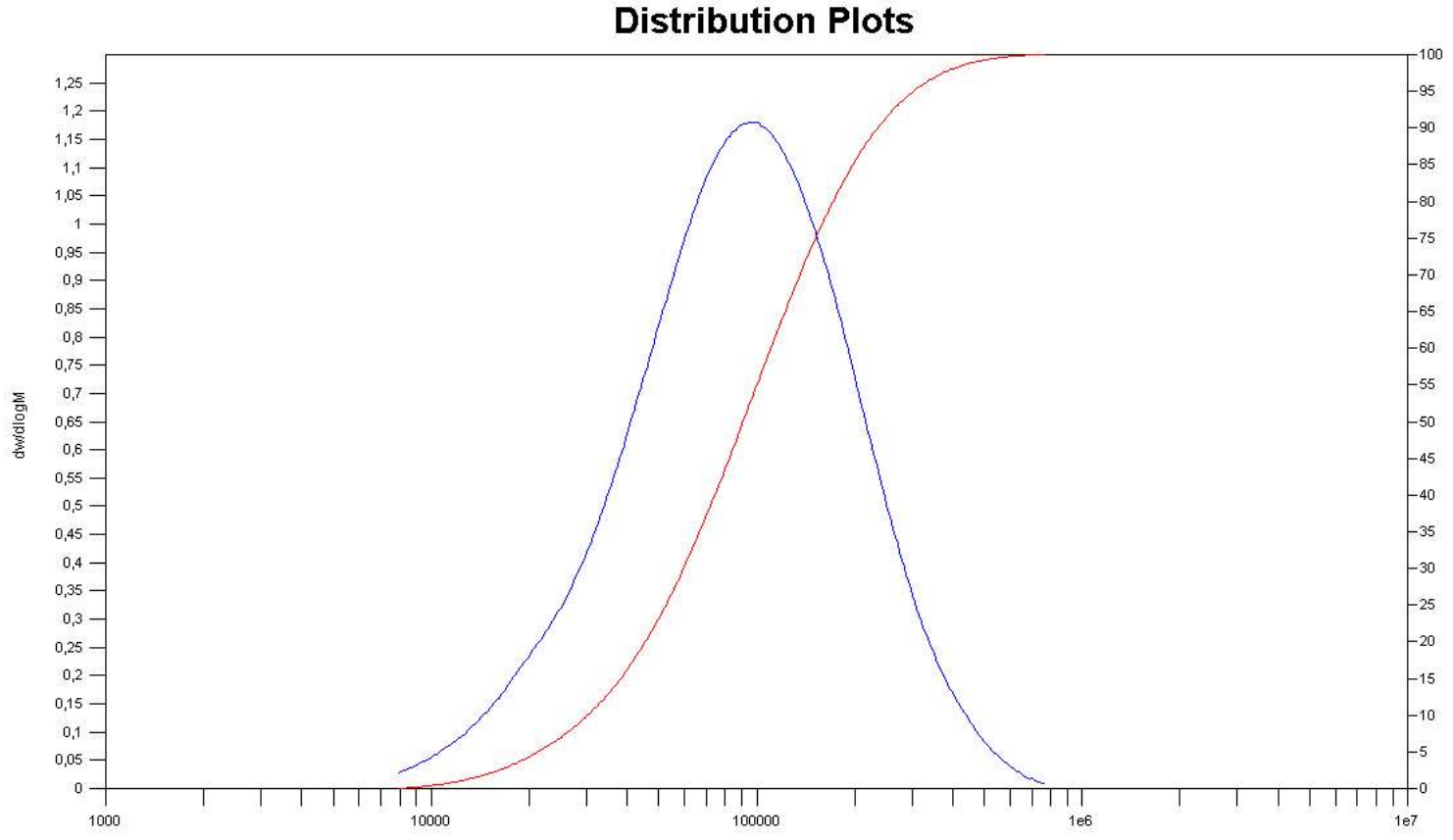

Figure S6. GPC curves showing molecular-weight retention: (a) syndiotactic 1,2-PBD used as starting polymer; (b) $>99 \%$ hydrogenated 1,2-PBD. Molecular weight determined versus polyethylene standards. 